\title{
Efectos de Trichoderma asperellum en la estimulación del crecimiento en chile dulce (Capsicum annum) variedad Nathalie en ambientes protegidos Effects of Trichoderma asperellum on growth stimulation in sweet pepper (Capsicum annum) variety Nathalie in greenhouse
}

\author{
Jaime Brenes-Madriz ${ }^{1}$, Claudia Zúñiga-Vega², \\ Marvin Villalobos-Araya ${ }^{3}$, Cristian Zúñiga-Poveda ${ }^{4}$, \\ William Rivera-Méndez ${ }^{5}$
}

Brenes-Madriz, J; Zúñiga-Vega, C; Villalobos-Araya, M; Zúñiga-Poveda, C; Rivera-Méndez, W. Efectos de Trichoderma asperellum en la estimulación del crecimiento en chile dulce (Capsicum annum) variedad Nathalie en ambientes protegidos. Tecnología en Marcha. Vol. 32-3. Julio-Setiembre 2019. Pág. 79-86.

DOI: https://doi.org/10.18845/tm.v32i3.4481

1 Ing. Agrónomo, Instituto Tecnológico de Costa Rica. Centro de Investigación en Biotecnología. Escuela de Biología. Costa Rica. Correo electrónico: jabrenes@tec.ac.cr. (iD https://orcid.org/0000-0003-2325-8808

2 Bióloga, Instituto Tecnológico de Costa Rica. Centro de Investigación en Biotecnología. Escuela de Biología. Costa Rica. Correo electrónico: czuniga@tec.ac.cr. (iD) https://orcid.org/0000-0001-7267-6788

3 Ing. Agrícola, Instituto Tecnológico de Costa Rica. Escuela de Ingeniería Agrícola. Costa Rica. Correo electrónico: mvillalobos@tec.ac.cr. (iD) https://orcid.org/0000-0002-1321-9971

4 Ing. Agrónomo, Instituto Tecnológico de Costa Rica. Escuela de Ingeniería Agrícola. Costa Rica. Correo electrónico: cristian.zuniga@ctasoluciones.com.

5 Ing. Biotecnología. Instituto Tecnológico de Costa Rica. Centro de Investigación en Biotecnología. Escuela de Biología. Costa Rica. Correo electrónico: wirivera@tec.ac.cr. (iD) https://orcid.org/0000-0002-2065-6264 


\title{
Palabras clave
}

Trichoderma asperellum; metabolitos secundarios; bio-filtrado; Capsicum annum; ambientes protegidos.

\section{Resumen}

La utilización de especies del hongo Trichoderma es de uso frecuente en el control biológico, donde inicialmente se aplicó por sus propiedades micoparásitas y antibióticas. Numerosas publicaciones han demostrado también su capacidad para colonizar las raíces y sus múltiples efectos favorables sobre los cultivos. Para Costa Rica no hay referencias que hagan mención al uso de filtrado de metabolitos secundarios para incrementar la producción de chile dulce. El objetivo de esta investigación consistió en comparar el efecto de filtrado de metabolitos secundarios presentes en un biofiltrado del hongo Trichoderma asperellum en la estimulación del crecimiento en chile dulce (Capsicum annum var Nathalie) en ambientes protegidos. Se evaluaron tratamientos a diferentes concentraciones de T. asperellum y de sus biofiltrados, en almácigos de esta hortaliza. Los efectos obtenidos con la aplicación de T. asperellum sobre la estimulación del crecimiento indican que la utilización de este biocontrolador tiene un impacto mayor en la etapa de almácigo. El tratamiento de T50 (T. asperellum al 50\%) resultó ser la mejor opción durante los dos años de análisis en la elongación de la raíz, además promovió un incremento en la biomasa de la raíz y el área foliar. Sin embargo, la utilización de este microorganismo en invernadero en la etapa de productividad, no presentó diferencias significativas entre los tratamientos, aunque se observó un buen desarrollo foliar de las plantas comparadas con el testigo.

\section{Keywords}

Trichoderma asperellum; secondary metabolites; cultured filtrate; Capsicum annum; protected environments.

\begin{abstract}
The use of Trichoderma fungus species is frequently used in biological control, where it was initially applied for its mycoparasitic and antibiotic properties. Numerous publications have also demonstrated its capacity to colonize the roots and their multiple favorable effects on crops. For Costa Rica there are no references that mention the use of secondary metabolites to increase production in sweet peppers. The objective of this research was to compare the effect of secondary metabolites present in a Trichoderma asperellum's cultured filtrated in the stimulation of growth in sweet pepper (Capsicum annum var Nathalie) in protected environments. Treatments were evaluated at different concentrations of $T$. asperellum and its biofiltrates, in sweet pepper seedlings of the variety. The effects obtained with the application of $T$. asperellum on the stimulation of growth indicate that the use of this biocontroller has a major impact on the nursery stage. The treatment of T50 (T. asperellum 50\%) was the best option during the two years of analysis in the elongation of the root, promoted an increase in the biomass of the root, besides it promoted an increase in the biomass of the root and the foliar area. However, the use of this microorganism in the greenhouse in the stage of productivity did not show significant differences between treatments, although a good foliar development of the plants compared with the control was observed.
\end{abstract}




\section{Introducción}

En Costa Rica, se ha incrementado el cultivo en ambientes protegidas. El 89\% de los invernaderos está destinado a la producción de hortalizas, con un 11\% dedicado al chile dulce. Esta forma de cultivar favorece la protección contra los factores climáticos, como los ocasionados por el cambio climático global y permite un manejo integrado de plagas y enfermedades, por lo que permite intensificar la producción. Por otra parte, el uso de tecnologías de sistemas de riego localizados, como el goteo, promueve una mejor eficiencia en el uso del agua y fertilizantes. Asimismo, en invernaderos se pueden aplicar técnicas que permiten una mayor producción por área comparada con el campo abierto, también los ciclos del cultivo se pueden extender, porque la planta no se encuentra sometida a cambios bruscos de temperatura, precipitación y exposición a fitopatógenos, lo que contribuye a mantener constante la calidad y la oferta [1,2].

La distancia de siembra juega un papel importante ya que es la que determina el mejor aprovechamiento del espacio dentro del invernadero; a campo abierto, el chile dulce se puede mantener a una distancia de $0.70 \mathrm{~m}-1.20 \mathrm{~m}$ entre hileras y entre plantas entre $0.40 \mathrm{~m}$ a $0.60 \mathrm{~m}$ con una producción de $8000 \mathrm{~kg}$ hasta $42000 \mathrm{~kg} / \mathrm{ha}$; mientras que en invernadero la producción podría aumentar con una distancia de $0.20 \mathrm{~m}-1.20 \mathrm{~m}$ entre hileras y a $0.20 \mathrm{~m}$ entre plantas en un rango de $30000 \mathrm{~kg}$ hasta $150000 \mathrm{~kg} / \mathrm{ha}$ [3]. Ramírez y Nienhuis [4] mencionan, que la producción de chile dulce en Costa Rica a campo abierto es en promedio de 20 frutos por planta, mientras que bajo invernadero puede alcanzar los 50 frutos por planta.

En Costa Rica, el uso de Trichoderma se ha generalizado, principalmente por su capacidad como antagonista de hongos $[5,6,7,8]$. Este hongo se localiza en todas las zonas climáticas del mundo, incluyendo la tundra, la Antártida y los trópicos. Numerosas publicaciones han demostrado también su gran capacidad para colonizar las raíces y los múltiples efectos favorables que ejerce sobre los cultivos $[9,10]$.

El género Trichoderma comprende muchas especies que producen una gran variedad de filtrado de metabolitos secundarios. Ya que es útil como biocontrolador no solo de hongos, sino también de bacterias y de virus. Estos metabolitos no son esenciales para la sobrevivencia del microorganismo, por lo que se denominan secundarios y cumplen diversas funciones. En la actualidad se está promoviendo su uso, ya que son más fáciles de manejar que los organismos completos, se necesitan menores cantidades para lograr el efecto deseado, presentan una vida útil más larga y además logran un efecto más directo y un estímulo mayor sobre el cultivo a tratar, por ser más específicos [11].

En algunas especies de este género, se han descubierto mecanismos moleculares que promueven una simbiosis con las plantas [12], lo cual incide en el crecimiento vegetal, la absorción de los nutrientes y la inducción de resistencia sistémica o localizada en las mismas, como consecuencia en parte de la colonización por el biocontrolador de la rizosfera y del sistema radicular, lo que trae como consecuencia un incremento en la productividad. Asimismo, se ha encontrado que proveen tolerancia al estrés abiótico (calor, frío, sal), solubilizan los nutrientes y hasta pueden actuar en procesos de biorremediación para degradar metales pesados y otros contaminantes ambientales [13].

Como el chile dulce es una hortaliza con un consumo per cápita anual de $1.6 \mathrm{~kg}$, donde se destinaron 1085 ha a la siembra de este cultivo y cuya producción bajo invernaderos se ha incrementado considerablemente en los últimos años $[3,14]$, se consideró importante, hacer una evaluación preliminar para comparar el efecto de filtrado de metabolitos secundarios presentes en un biofiltrado del hongo Trichoderma asperellum en la estimulación del crecimiento en chile dulce (Capsicum annum var Nathalie) en ambientes protegidos, con miras a disminuir los costos relacionados con el uso de agroquímicos. 


\section{Materiales y métodos}

\section{Etapa de almácigo}

Se establecieron almácigos de chile dulce, variedad Nathalie, en la empresa Villa Plant, los cuales fueron inoculados con T. asperellum. Las semillas se plantaron en bandejas plásticas con capacidad para 288 plantas; se llenaron con una mezcla de sustrato compuesto de 1/3 abono orgánico, 2/3 suelo y un complemento de granza de arroz a razón de $100 \mathrm{~g} / \mathrm{k}$ de mezcla, desinfectado previamente con vapor a $80^{\circ} \mathrm{C}$. El llenado de las bandejas con sustrato se realizó de forma manual y la siembra de las semillas por medio de una sembradora mecánica. Posteriormente, se cubrió con una capa de sustrato y se le aplicó cada uno de los tratamientos. Las bandejas cultivadas se colocaron en cámaras de germinación a una temperatura de $23^{\circ} \mathrm{C}$ y una humedad relativa del 96\%, para asegurar una buena geminación; la inoculación y rotulación del almácigo se realizó al día siguiente de sembradas las semillas.

Los tratamientos evaluados fueron:

1. Testigo absoluto, en el cual no se utilizó aditivo para la protección radicular

2. T. asperellum al $50 \%$ (55 g de un preparado de T. asperellum por $3.6 \mathrm{~L}$ de agua cultivado en arroz).

3. T. asperellum al 100\% (110 g de un preparado de T. asperellum por $3.6 \mathrm{~L}$ de agua, cultivado en arroz).

4. Tratamiento con agroquímicos a base de Vitavax®) al 100\% (dosis de $8.5 \mathrm{~g}$ del producto disuelto en $3.6 \mathrm{~L}$ de agua).

Las aplicaciones de los diferentes tratamientos se realizaron cada 10 días. Las plántulas se trasladaron al invernadero a los 15 días, donde se continuó con las aplicaciones de los distintos tratamientos. Se hicieron mediciones de peso seco de las raíces y el área foliar, así como de la longitud de la raíz, para el análisis de almácigos. El experimento se repitió durante el siguiente año y se añadieron dos tratamientos más que fueron: la aplicación de un biofiltrado de metabolitos de T. asperellum al 100\% fue el quinto tratamiento y la aplicación de un biofiltrado de metabolitos de T. asperellum al 50\% el sexto. Se hicieron mediciones del peso seco del área foliar, la longitud de la raíz y el peso seco de la raíz.

\section{Etapa de invernadero}

Para la etapa de campo, las plántulas de chile se sembraron en un invernadero, perteneciente a la Escuela de Ingeniería Agrícola del Instituto Tecnológico de Costa Rica (TEC). Se evaluaron seis filas con 30 plantas cada una, las cuales se marcaron con cintas de colores y a cada fila se le aplicó un tratamiento. Para la preparación de filtrado de metabolitos secundarios al 100\% se utilizaron $4.5 \mathrm{ml}$ del biofiltrado, que se mezcló en un beaker con $900 \mathrm{ml}$ de agua y se aforó. Para la solución al 50\% se utilizó $2.25 \mathrm{ml}$ del preparado anterior, los cuales se obtuvieron agregando estos a un beaker con $900 \mathrm{ml}$ de agua y se aforó a un litro. Las aplicaciones de cada tratamiento se realizaron cada 10 días, hasta concluir con el período correspondiente, según el desarrollo del cultivo.

El detalle de los tratamientos evaluados, en un diseño de bloques completos al azar fue:

1. T. asperellum al $100 \%$ (110 g de un preparado de T. asperellum por $3.6 \mathrm{~L}$ de agua, cultivado en arroz).

2. T. asperellum al 50\% (55 g de un preparado de T. asperellum por $3.6 \mathrm{~L}$ de agua cultivado en arroz). 
3. Tratamiento con agroquímicos a base de Vitavax $®$ como protector radicular en una dosis de $8.5 \mathrm{~g}$ del producto disuelto en $3.6 \mathrm{~L}$ de agua.

4. Aplicación de un biofiltrado de metabolitos de T. asperellum al 100\%.

5. Aplicación de un biofiltrado de metabolitos de T. asperellum al 50\%.

6. Testigo absoluto, en el cual no se utilizó ningún aditivo para la protección radicular.

Se hicieron mediciones de la cantidad de frutos producidos por tratamiento durante dos meses, y se evaluó la calidad del producto en primera, segunda y tercera, además se determinó el peso de lo producido en cada tratamiento.

\section{Resultados y discusión}

\section{Etapa de almácigo}

En el cuadro 1 se presentan los resultados obtenidos en cada uno de los tratamientos y las variables analizadas. Con el tratamiento T50 se logró el mayor peso aéreo seco. Para las variables de longitud de la raíz y peso seco de la raíz, T50 y T100 fueron los tratamientos que presentaron el mejor comportamiento. De lo anterior, se podría recomendar el uso de T50, porque produce mayores ventajas, tanto a nivel de raíz, como de parte aérea y es más económico. El tratamiento con agroquímicos no se debe recomendar, porque el crecimiento que promueve no es correspondido por un incremento en la biomasa de la planta. Para el segundo año, los tratamientos con Trichoderma al 100\% y 50\%, junto con el Testigo y el biofiltrado al 50\% tuvieron el mismo impacto sobre la elongación de la raíz. Sin embargo, si se considera el peso seco, el mayor aumento en la biomasa de la raíz se logró con Trichoderma al 50\%.

T50 también fue el mejor tratamiento en lo que se refiere al peso seco. De lo anterior, se podría recomendar la utilización de T50, por ser más económico que T100 y más fácil de preparar que B50 (Cuadro 1). Mientras que el tratamiento con agroquímicos no aparece en ninguno de los mejores tratamientos obtenidos, por lo que es importante reconsiderar su utilización, tomando en cuenta el parámetro de costo/beneficio.

Cuadro 1. Comparación entre las variables analizadas y los tratamientos aplicados. Año 1 y 2

\begin{tabular}{|c|c|c|c|c|}
\hline Variable & $\begin{array}{c}\text { Año } 1 \\
\text { Mejor tratamiento y } \\
\text { media }\end{array}$ & $P^{*}$ & $\begin{array}{c}\text { Año } 2 \\
\text { Mejor tratamiento y } \\
\text { media }\end{array}$ & $P^{*}$ \\
\hline $\begin{array}{l}\text { Peso seco } \\
\text { área foliar }\end{array}$ & $\begin{array}{ll}\text { T50 } & 311.400 \\
\text { Test } & 280.700\end{array}$ & $\begin{array}{l}\text { Kruskall Wallis } \\
P=1.916 e-05\end{array}$ & $\begin{array}{ll}\text { B50 } & 163.3333 \\
\text { T50 } & 143.0667\end{array}$ & $\begin{array}{c}\text { Kruskall } \\
P=6.289 e-13\end{array}$ \\
\hline Longitud raíz & $\begin{array}{ll}\text { T50 } & 118.53675 \\
\text { T100 } & 112.27625\end{array}$ & $\begin{array}{l}\text { Kruskall Wallis } \\
p=0.0002111\end{array}$ & $\begin{array}{cc}\text { Test } & 4.706443 \\
\text { B50 } & 4.691537\end{array}$ & $\begin{array}{c}\text { ANOVA } \\
P=0.000125\end{array}$ \\
\hline Peso seco raíz & $\begin{array}{ll}\text { T50 } & 171.300 \\
\text { T100 } & 162.925\end{array}$ & $\begin{array}{l}\text { Kruskall Wallis } \\
P=0.0002111\end{array}$ & $\begin{array}{cc}\text { T50 } & 69.93333 \\
\text { B50 } & 64.0666\end{array}$ & $\begin{array}{c}\text { Kruskall } \\
P=1.436 e-07\end{array}$ \\
\hline
\end{tabular}

${ }^{\star} P$ : prueba de significancia. Se aplicó Kruskall wallis o ANOVA según la Normalidad y Homocedasticidad de la muestra

\section{Etapa de invernadero}

En el cuadro 2 se observa la producción obtenida durante ocho semanas de chile dulce por tratamiento, según calidad y cantidad. En el cuadro 3, se presenta el peso total en kg de los chiles dulces cosechados por tratamiento. 
Cuadro 2. Producción de chile dulce por tratamiento, según calidad y cantidad.

\begin{tabular}{|c|c|c|c|c|c|c|c|c|c|c|c|c|c|c|c|c|c|}
\hline \multicolumn{18}{|c|}{ Tratamientos } \\
\hline \multicolumn{3}{|c|}{1} & \multicolumn{3}{|c|}{2} & \multicolumn{3}{|c|}{3} & \multicolumn{3}{|c|}{4} & \multicolumn{3}{|c|}{5} & \multicolumn{3}{|c|}{6} \\
\hline $1 a$ & $2 a$ & $3 a$ & $1 a$ & $2 a$ & $3 a$ & $1 a$ & $2 a$ & $3 a$ & $1 a$ & $2 a$ & $3 a$ & $1 a$ & $2 a$ & $3 a$ & $1 \mathrm{a}$ & $2 a$ & $3 a$ \\
\hline 103 & 29 & 21 & 101 & 35 & 22 & 75 & 44 & 21 & 90 & 33 & 26 & 102 & 45 & 17 & 90 & 30 & 29 \\
\hline \multicolumn{3}{|c|}{ Total 153} & \multicolumn{3}{|c|}{ Total 158} & \multicolumn{3}{|c|}{ Total 140} & \multicolumn{3}{|c|}{ Total 149} & \multicolumn{3}{|c|}{ Total 164} & \multicolumn{3}{|c|}{ Total 149} \\
\hline
\end{tabular}

Cuadro 3. Peso total $(\mathrm{kg})$ de los chiles cosechado por tratamiento

\begin{tabular}{|c|c|c|c|c|c|c|}
\hline Tratamiento & T1 & T2 & T3 & T4 & T5 & T6 \\
\hline & 20,55 & 21,38 & 18,00 & 18,63 & 21,61 & 19,63 \\
\hline
\end{tabular}

El análisis estadístico realizado sobre los datos de rendimiento en invernadero no mostró diferencias significativas para ningún tratamiento con respecto al control ( $p>0,05)$. Lo mismo ocurrió para las variables de primera, segunda y tercera calidad. En la figura 1 se detalla el comportamiento obtenido en el rendimiento de los chiles dulces, durante 8 semanas por tratamiento. Y en la figura 2 el rendimiento de chiles dulces en $\mathrm{kg}$ según tratamiento aplicado y calidad del fruto.

25

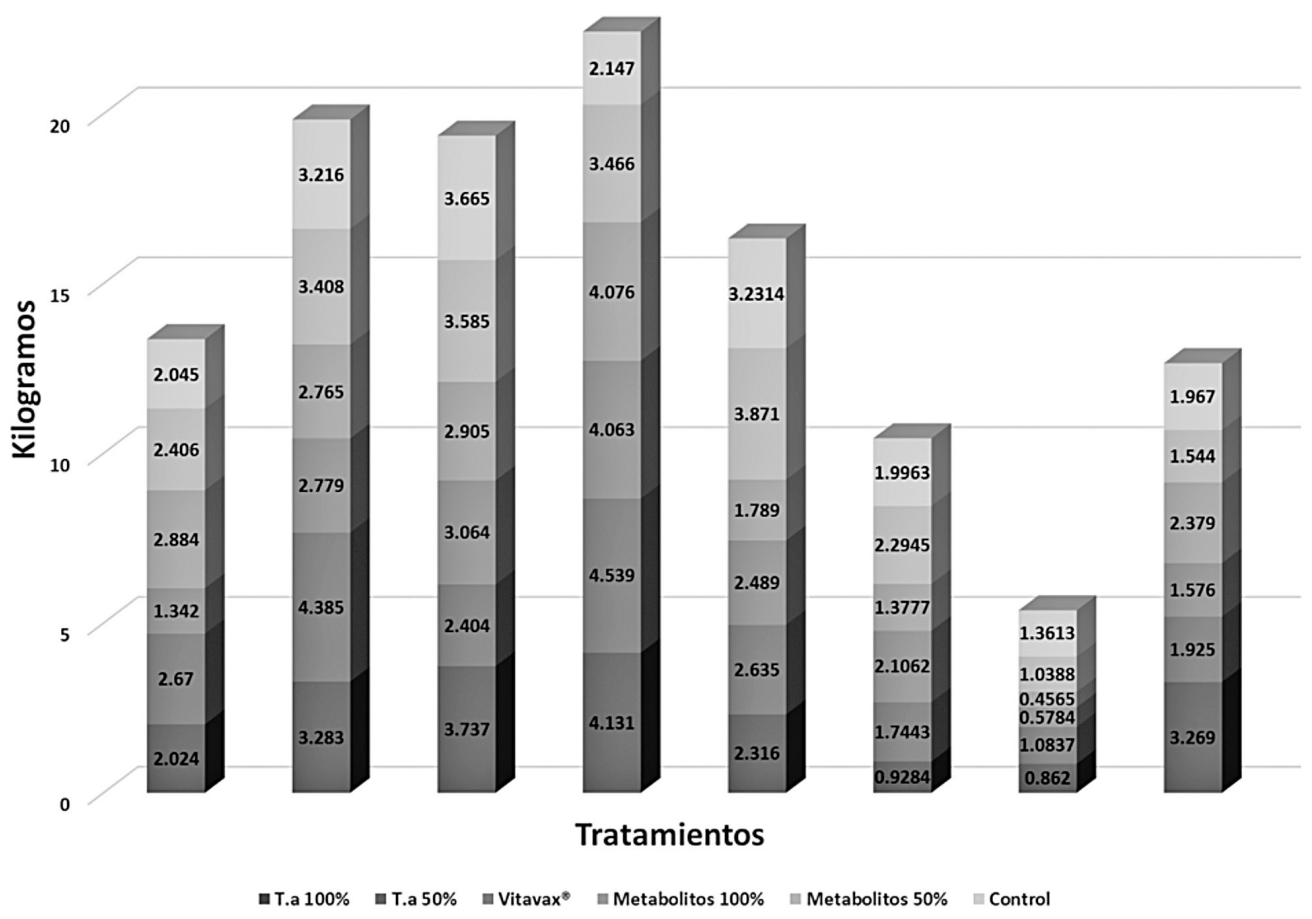

Figura 1. Rendimiento en kilogramos de chile dulce en ocho fechas de muestreo según tratamiento. 


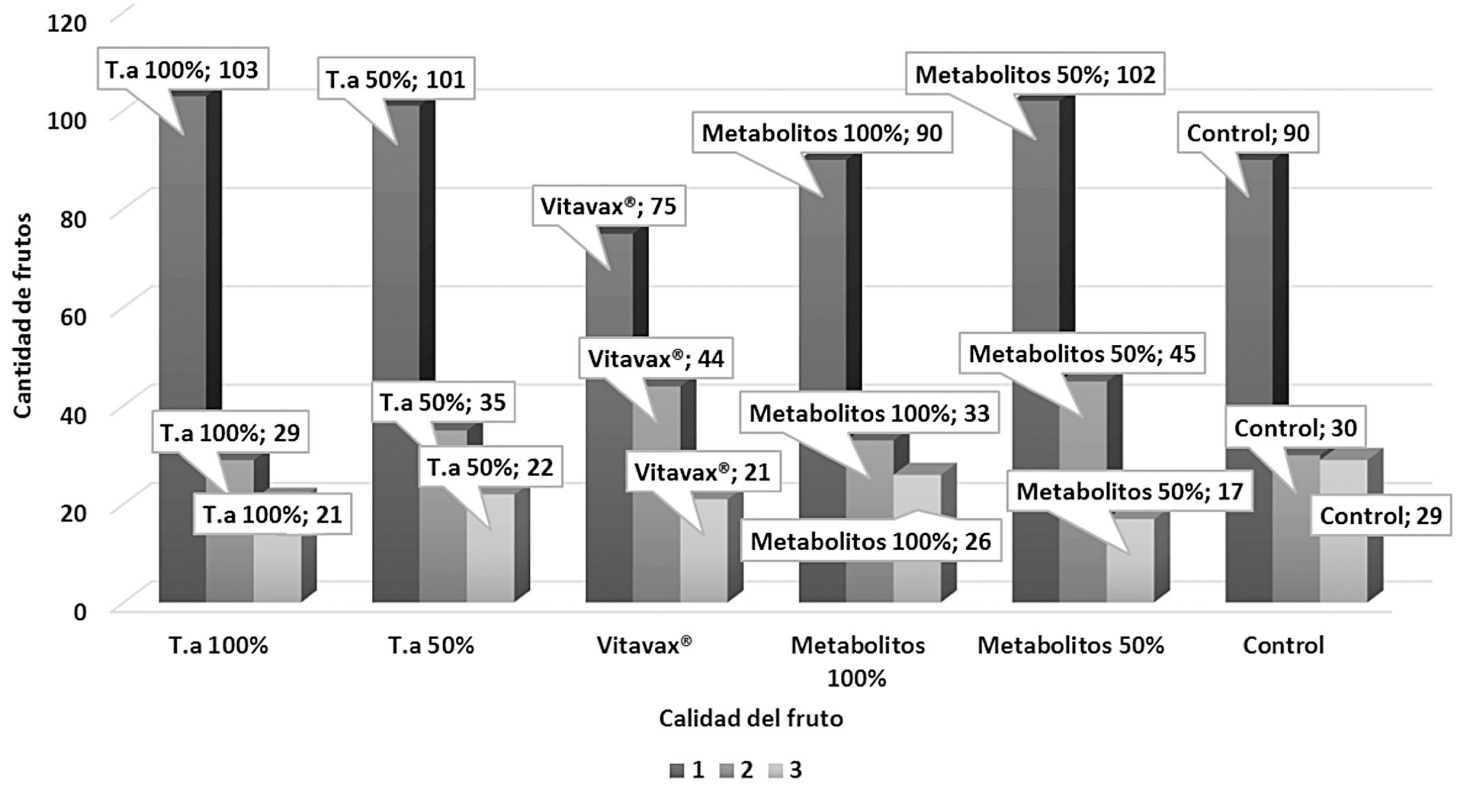

Figura 2. Rendimiento de chiles dulces en kg según tratamiento aplicado y calidad del fruto

\section{Conclusiones}

Los efectos obtenidos con la aplicación de T. asperellum sobre la estimulación del crecimiento en chile dulce (Capsicum annum) variedad Nathalie en ambientes protegidos indican que la utilización de este biocontrolador tiene un impacto mayor en la etapa de almácigo. El tratamiento de T50 resultó ser la mejor opción durante los dos años de análisis en la elongación de la raíz, además promovió un incremento en la biomasa de la raíz y el área foliar. Sin embargo, la utilización de este microorganismo en la etapa de invernadero en la productividad, no presentó diferencias significativas entre los tratamientos, aunque se observó un buen desarrollo foliar de las plantas comparadas con el testigo.

\section{Agradecimientos}

Este proyecto contó con el financiamiento de la Vicerrectoría de Investigación y Extensión del Instituto Tecnológico de Costa Rica.

\section{Referencias}

[1] C. Méndez and F. Marín, "El concepto de agricultura protegida para el Trópico latinoamericano". Boletín ProNAP vol. 9, pp. 2-12, 2015.

[2] J. Monge, "Evaluación de 60 genotipos de tomate (Lycopersicon esculentum Mill.) cultivados bajo invernadero en Costa Rica". Intersedes, vol. 16, pp. 88-122, 2015.

[3] M. Carrillo et al., "Agrocadena regional cultivo chile dulce" Ministerio de Agricultura y Ganadería. Alajuela, Costa Rica. Disponible: http://www.mag.go.cr/bibliotecavirtual/a00069.pdf , 2007.

[4] C. Ramírez and J. Nienhuis, "Cultivo protegido de hortalizas en Costa Rica". Tecnología en Marcha, vol. 25, pp. 10-20, 2012.

[5] L. Alvarado-Marchena and W. Rivera-Méndez, "Molecular Identification of Trichoderma spp. in garlic and onion fields and in vitro antagonism trials on Sclerotium cepivorum". Rev Bras Cienc Solo, vol 40:e0150454, 2016. 
[6] K. Astorga-Quirós et al., "Evaluación del antagonismo de Trichoderma sp. y Bacillus subtilis contra tres patógenos del ajo". Tecnología en Marcha, vol. 27, pp. 82-91, 2014.

[7] W. Bettiol et al. (Eds.), "Control biológico de enfermedades de plantas en América Latina y el Caribe". ISBN: 978-9974-0-1091-8, 2014.

[8] J. Brenes et al. , "Cultivo del ajo en Costa Rica". EUNED. ISBN 978-9968-31-958-4, 100p, 2013.

[9] G. E. Harman, "Overview of mechanisms and uses of Trichoderma spp". Phytopathology, vol. 96, pp. 190-194, 2006.

[10] I. Samolski et al., "The gid74 gene from Trichoderma harzianum has a role in root architecture and plant biofertilization". Microbiology, vol. 158, pp.129-138, 2012.

[11] Keswani et al., "Unraveling the efficient applications of secondary metabolites of various Trichoderma spp". Appl Microbiol Biotechnol, vol. 98, pp. 533-544, 2014.

[12] M. Lorito et al., "Translational Research on Trichoderma: From Omics to the Field". Annu Rev Phytopathol, vol. 48, pp. 395-417, 2010.

[13] P.K. Mukherjee et al., "Trichoderma Research in the Genome Era". Annu. Rev. Phytopathol. vol. 51, pp. 105-129, 2013.

[14] Costa Rica, "VI Censo Nacional Agropecuario 2014, Resultados Generales". Instituto Nacional de Estadística y Censos, p. 76, 2015. 